\title{
Nonlinear Free Vibration for Viscoelastic Moderately Thick Laminated Composite Plates with Damage Evolution
}

\author{
Y. F. Zheng and L. Q. Deng \\ College of Civil Engineering, Fuzhou University, Fuzhou 350108, China \\ Correspondence should be addressed to Y. F. Zheng, zheng-yufang@163.com
}

Received 22 December 2009; Accepted 20 March 2010

Academic Editor: Carlo Cattani

Copyright (C) 2010 Y. F. Zheng and L. Q. Deng. This is an open access article distributed under the Creative Commons Attribution License, which permits unrestricted use, distribution, and reproduction in any medium, provided the original work is properly cited.

The nonlinear free vibration for viscoelastic cross-ply moderately thick laminated composite plates under considering transverse shear deformation and damage effect is investigated. Based on the Timoshenko-Mindlin theory, strain-equivalence hypothesis, and Boltzmann superposition principle, the nonlinear free vibration governing equations for viscoelastic moderately thick laminated plates with damage are established and solved by the Galerkin method, Simpson integration, Newton-Cotes, Newmark, and iterative methods. In the numerical results, the effects of transverse shear, material viscoelasticity, span-thickness ratio, aspect ratio, and damage effect on the nonlinear free vibrating frequency of the viscoelastic cross-ply moderately thick laminated plates are discussed.

\section{Introduction}

The structure will present the resonance phenomenon when the external excitation frequency is near to a certain natural frequency of the structure during the service life of the structures. Structure destructions caused by the resonance are prevalent in the practical engineering. The damage will emerge in the viscoelastic composite structures during the process of vibration and lead to the change of the dynamic behavior. When the damage develops, the structure will probably enter into the resonant state. As soon as the resonance appears, the stress values in the structure will increase, which will cause the development of the damage accelerate. Therefore, it is a very important research field to investigate the nonlinear dynamic behavior of viscoelastic laminated plates with damage effect.

Extensive studies have been made in dynamics of viscoelastic homogeneous structures. On the basis of the linear theory and the concept of the Lyapunov exponents, Aboudi and Cederbaum [1] investigated the dynamic stability of viscoelastic rectangular plates. 
Librescu and Chandiramani [2] analyzed the dynamic stability of transversely isotropic viscoelastic plates. Sun and Zhang [3] investigated the chaotic behaviors of viscoelastic rectangular plates subjected to an in-plane periodic load and pointed out that the stability of the structure could be increased by adjusting the material parameters. Chen et al. [4] analyzed the steady-state response of the parametrically excited axially moving string constituted by the Boltzmann superposition principle. T. W. Kim and J. H. Kim [5] applied finite element analysis and the method of multiple scales to investigate the nonlinear vibrating frequency of viscoelastic laminated plates. Yu and Huang [6] presented a mathematical model for the vibration of a three-layered sandwich circular plate with viscoelastic core and discussed the effect of viscoelasticity on the frequency and amplitude. Relatively, few works have been devoted to study the effects of local damage and defects on the static and dynamic behavior of plates. Prabhakara and Datta $[7,8]$ analyzed the effect of the structural flaw on the natural frequency and buckling load of elastic plates subjected to a uniform in-plane load. Laura and Gutierrez [9] presented the linear fundamental frequency of transverse vibration for a damaged circular annular plate. However, the materials of the composite laminated plates have the property of viscoelasticity with the apparent creep phenomenon and relaxation characteristic, so it is very necessary to examine the influences of the damage effect on the nonlinear dynamics of viscoelastic laminated plates. Sheng and Cheng [10] used the history curve, phase trajectory diagram, Poincare map, bifurcation figure, and power spectrum to analyze the nonlinear dynamical properties of viscoelastic thick plate with damage. Fu et al. $[11,12]$ studied the nonlinear dynamic response of viscoelastic composite plate with transverse matrix cracks based on Schapery's 3D constitutive relationship. To author's work, Zheng and $\mathrm{Fu}$ [13] have studied the effect of local damage on the bifurcation and chaos of viscoelastic isotropic plates, and the nonlinear dynamic properties of viscoelastic isotropic plates and laminated plates with considering damage evolution [14-16].

In the present study, the nonlinear free vibration equations of the viscoelastic cross-ply moderately thick laminated composite plates with damage effect are established by applying Timoshenko-Mindlin theory, strain equivalence hypothesis, and Boltzmann superposition principle. By employing the standard linear solid model to express the viscoelastic material properties, Kachanov's approach to describe the damage evolution, and using the Galerkin method, Simpson integration, Newton-Cotes, Newmark method, and iterative procedure, the solutions of the problem are obtained. Numerical results are presented for different parameters.

\section{Basic Equations}

Consider a viscoelastic cross-ply rectangular plate having length $a$ in the $x$ direction, width $b$ in the $y$ direction, and thickness $h$ in the $z$ direction. The middle plane of the undeformed plate contains the $x, y$ axes and the origin of the coordinate system is taken at the upper left corner of the plate. Based on Timoshenko-Mindlin kinematic hypotheses taking into account the transverse normal deformation, the displacement components $u_{1}, u_{2}$, and $u_{3}$ that include the effect of transverse shear deformation may be described by the following expressions [17]:

$$
\begin{gathered}
u_{1}(x, y, z, t)=u(x, y, t)+z \varphi(x, y, t), \\
u_{2}(x, y, z, t)=v(x, y, t)+z \psi(x, y, t), \\
u_{3}(x, y, z, t)=w(x, y, t),
\end{gathered}
$$


where $t$ is the time, $u, v$, and $w$ are the values of $u_{1}, u_{2}$, and $u_{3}$ at the middle surface, and $\varphi$ and $\psi$ are rotation angles of the normal to the middle surface in the $x z$ and $y z$ planes, respectively. The nonlinear strain-displacement relationship can be written as

$$
\begin{gathered}
\varepsilon_{x}=\varepsilon_{x}^{0}+z \kappa_{x}, \quad \varepsilon_{y}=\varepsilon_{y}^{0}+z \kappa_{y}, \quad \varepsilon_{x y}=\varepsilon_{x y}^{0}+z \kappa_{x y}, \\
\varepsilon_{x z}=\varphi+w_{, x}, \quad \varepsilon_{y z}=\psi+w_{, y}
\end{gathered}
$$

where a comma denotes partial differentiation with respect to the corresponding coordinates and where

$$
\begin{array}{cl}
\varepsilon_{x}^{0}=u_{, x}+\frac{1}{2} w_{, x}^{2}, & \mathcal{\kappa}_{x}=\varphi, x, \\
\varepsilon_{y}^{0}=v_{, y}+\frac{1}{2} w_{, y}^{2}, & \kappa_{y}=\psi, y, \\
\varepsilon_{x y}^{0}=u_{, y}+v_{, x}+w_{, x} w_{, y}, & \kappa_{x y}=\varphi_{, y}+\psi, x .
\end{array}
$$

By applying the loading equivalent principle and assuming that the internal forces acting on any damaged section are the same as the ones before damage, the relationship between the effective stresses $\widetilde{\sigma}_{i j}$ and the Cauchy stresses $\sigma_{i j}$ is given as [18]

$$
\left[\begin{array}{c}
\tilde{\sigma}_{x} \\
\tilde{\sigma}_{y} \\
\tilde{\sigma}_{x y} \\
\tilde{\sigma}_{x z} \\
\tilde{\sigma}_{y z}
\end{array}\right]=\left[\begin{array}{ccccc}
\frac{1}{1-D_{1}} & 0 & 0 & 0 & 0 \\
0 & \frac{1}{1-D_{2}} & 0 & 0 & 0 \\
0 & 0 & \frac{1}{\sqrt{\left(1-D_{1}\right)\left(1-D_{2}\right)}} & 0 & 0 \\
0 & 0 & 0 & \frac{1}{\sqrt{1-D_{1}}} & 0 \\
0 & 0 & 0 & 0 & \frac{1}{\sqrt{1-D_{2}}}
\end{array}\right]\left[\begin{array}{c}
\sigma_{x} \\
\sigma_{y} \\
\sigma_{x y} \\
\sigma_{x z} \\
\sigma_{y z}
\end{array}\right] \text {, }
$$

where the anisotropic damage variables $D_{1}$ and $D_{2}$ are similarly defined as in $[15,16]$.

The above relation is expressed as follows:

$$
\widetilde{\sigma}=\Lambda \sigma
$$

Employing the strain energy equivalence principle [19] and Boltzmann superposition principle [20], the stress-strain constitutive equations of the coupled viscoelastic/damage cross-ply laminated plates for the $k$ th layer can be obtained in the following form:

$$
\boldsymbol{\sigma}^{(k)}(t)=T^{(k)^{\mathrm{T}}} \boldsymbol{\Lambda}^{(k)^{-1}} \mathbf{Q}^{(k)}(0) \boldsymbol{\Lambda}^{(k)^{-1}} T^{(k)} \varepsilon^{(k)}(t)+T^{(k)^{\mathrm{T}}} \boldsymbol{\Lambda}^{(k)^{-1}} \int_{0}^{t} \dot{\mathbf{Q}}^{(k)}(t-\tau) \boldsymbol{\Lambda}^{(k)^{-1}} T^{(k)} \mathcal{\varepsilon}^{(k)}(\tau) \mathrm{d} \tau
$$

in which $\mathbf{Q}^{(k)}(t)$ is the time-dependent relaxation function and $\mathbf{Q}^{(k)}(0)$ is the initial Young's modulus of materials for the $k$ th layer. For orthotropic viscoelastic materials and considering 
that Poisson ratio contains constant, $T^{(k)}$ is the strain transformation relation for the $k$ th layer, having

$$
\begin{gathered}
\mathbf{Q}^{(k)}(t)=\left[\begin{array}{ccccc}
\frac{E_{1}^{(k)}(t)}{1-v_{12}^{(k)} v_{21}^{(k)}} & \frac{v_{12}^{(k)} E_{2}^{(k)}(t)}{1-v_{12}^{(k)} v_{21}^{(k)}} & 0 & 0 & 0 \\
\frac{v_{12}^{(k)} E_{2}^{(k)}(t)}{1-v_{12}^{(k)} v_{21}^{(k)}} & \frac{E_{2}^{(k)}(t)}{1-v_{12}^{(k)} v_{21}^{(k)}} & 0 & 0 & 0 \\
0 & 0 & G_{12}^{(k)}(t) & 0 & 0 \\
0 & 0 & 0 & G_{13}^{(k)}(t) & 0 \\
0 & 0 & 0 & 0 & G_{23}^{(k)}(t)
\end{array}\right], \\
\mathbf{T}^{(k)}=\left[\begin{array}{ccccc}
\cos ^{2} \theta_{k} & \sin ^{2} \theta_{k} & \sin \theta_{k} \cos \theta_{k} & 0 & 0 \\
\sin ^{2} \theta_{k} & \cos ^{2} \theta_{k} & -\sin \theta_{k} \cos \theta_{k} & 0 & 0 \\
-2 \sin \theta_{k} \cos \theta_{k} & 2 \sin \theta_{k} \cos \theta_{k} & \cos 2 \theta_{k} & 0 & 0 \\
0 & 0 & 0 & \cos \theta_{k} & \sin \theta_{k} \\
0 & 0 & 0 & -\sin \theta_{k} & \cos \theta_{k}
\end{array}\right],
\end{gathered}
$$

where $c=\cos \theta_{k}$ and $s=\sin \theta_{k} . \theta_{k}$ is the angle measured from the fibre direction to coordinate axis $o x$ for the $k$ th layer; here $\theta_{k}=0$ or $90^{\circ}$

For simplification, (2.6) is rewritten as

$$
\begin{aligned}
& \boldsymbol{\sigma}_{1}^{(k)}=\mathbf{Q}^{d(k)} \otimes \varepsilon_{1}^{(k)}, \\
& \boldsymbol{\sigma}_{2}^{(k)}=\mathbf{Q}_{*}^{d(k)} \otimes \varepsilon_{2}^{(k)},
\end{aligned}
$$

where the symbol $(\otimes)$ is the Stieltjes convolution operation symbol, which is defined as

$$
\begin{gathered}
f \otimes g=\int_{-\infty}^{t} f(t-\tau) \mathrm{d} g(\tau)=f(0) g(t)+\int_{0}^{t} \dot{f}(t-\tau) g(\tau) \mathrm{d} \tau, \\
\sigma_{1}^{(k)}=\left\{\sigma_{x}^{(k)}, \sigma_{y}^{(k)}, \sigma_{x y}^{(k)}\right\}^{T}, \quad \sigma_{2}^{(k)}=\left\{\sigma_{x z}^{(k)}, \sigma_{y z}^{(k)}\right\}^{T}, \\
\varepsilon_{1}^{(k)}=\left\{R_{1}^{(k)} \varepsilon_{x}, R_{2}^{(k)} \varepsilon_{x}, R_{3}^{(k)} \varepsilon_{x}, R_{1}^{(k)} \varepsilon_{y}, R_{2}^{(k)} \varepsilon_{y}, R_{3}^{(k)} \varepsilon_{y}, R_{1}^{(k)} \varepsilon_{x y}, R_{2}^{(k)} \varepsilon_{x y}, R_{3}^{(k)} \varepsilon_{x y}\right\}^{T}, \\
\varepsilon_{2}^{(k)}=\left\{R_{4}^{(k)} \varepsilon_{x z}, R_{5}^{(k)} \varepsilon_{x z}, R_{4}^{(k)} \varepsilon_{y z}, R_{5}^{(k)} \varepsilon_{y z}\right\}^{T}, \\
\mathbf{Q}^{d(k)}=\left[Q_{i j}^{d(k)}\right], \quad(i=1,2,3, j=1,2, \ldots, 9), \quad Q_{*}^{d(k)}=\left[Q_{i j}^{d(k)}\right], \quad(i=4,5, j=1,2,3,4), \\
R_{1}^{(k)}=1-D_{1}^{(k)}, \quad R_{2}^{(k)}=1-D_{2}^{(k)}, \quad R_{3}^{(k)}=\sqrt{\left(1-D_{1}^{(k)}\right)\left(1-D_{2}^{(k)}\right)}, \\
R_{4}^{(k)}=\sqrt{\left(1-D_{1}^{(k)}\right)}, \quad R_{5}^{(k)}=\sqrt{\left(1-D_{2}^{(k)}\right)} .
\end{gathered}
$$


The nonzero elements in $\mathbf{Q}^{d(k)}$ and $\mathbf{Q}_{*}^{d(k)}$ are given as

$$
\begin{aligned}
& Q_{11}^{d(k)}=c^{4} R_{1}^{(k)} Q_{11}^{(k)} ， \quad Q_{12}^{d(k)}=s^{4} R_{2}^{(k)} Q_{22}^{(k)} ， \quad Q_{14}^{d(k)}=s^{4} R_{2}^{(k)} Q_{12}^{(k)} ， \quad Q_{15}^{d(k)}=c^{4} R_{1}^{(k)} Q_{12}^{(k)} ， \\
& Q_{21}^{d(k)}=c^{4} R_{2}^{(k)} Q_{12}^{(k)}, \quad Q_{22}^{d(k)}=s^{4} R_{1}^{(k)} Q_{12}^{(k)}, \quad Q_{24}^{d(k)}=s^{4} R_{1}^{(k)} Q_{11}^{(k)}, \quad Q_{25}^{d(k)}=c^{4} R_{2}^{(k)} Q_{22}^{(k)} \text { ， } \\
& Q_{39}^{d(k)}=\left(c^{2}-s^{2}\right)^{2} R_{3}^{(k)} Q_{66}^{(k)}, \quad Q_{41}^{d(k)}=c^{2} R_{4}^{(k)} Q_{44}^{(k)}, \quad Q_{42}^{d(k)}=s^{2} R_{5}^{(k)} Q_{55}^{(k)}, \\
& Q_{53}^{d(k)}=s^{2} R_{4}^{(k)} Q_{44}^{(k)}, \quad Q_{54}^{d(k)}=c^{2} R_{5}^{(k)} Q_{55}^{(k)} .
\end{aligned}
$$

As the classical plate theory, the stress resultants and couples are defined by

$$
\begin{gathered}
{\left[N_{x}, N_{y}, N_{x y}\right]=\int_{-h / 2}^{h / 2}\left[\sigma_{x}^{(k)}, \sigma_{y}^{(k)}, \sigma_{x y}^{(k)}\right] \mathrm{d} z} \\
{\left[Q_{x}, Q_{y}\right]=\int_{-h / 2}^{h / 2}\left[k_{s} \sigma_{x z}^{(k)}, k_{s} \sigma_{y z}^{(k)}\right] \mathrm{d} z} \\
{\left[M_{x}, M_{y}, M_{x y}\right]=\int_{-h / 2}^{h / 2}\left[\sigma_{x}^{(k)}, \sigma_{y}^{(k)}, \sigma_{x y}^{(k)}\right] z \mathrm{~d},}
\end{gathered}
$$

where, $N_{x}, N_{y}$, and $N_{x y}$ are the membrane stress resultants per unit length, $Q_{x}$ and $Q_{y}$ are the transverse shear stress resultants per unit length, $M_{x}, M_{y}$, and $M_{x y}$ are the bending and twisting moments per unit length, and $k_{s}$ is the shear correction factor taking $k_{s}=5 / 6$. Substituting (2.8) into (2.11), we can obtain

$$
\begin{gathered}
\left\{\begin{array}{l}
\mathbf{N} \\
\mathbf{M}
\end{array}\right\}=\sum_{k=1}^{n}\left[\begin{array}{ll}
\mathbf{A}^{(k)} & \mathbf{B}^{(k)} \\
\mathbf{B}^{(k)} & \mathbf{D}^{(k)}
\end{array}\right] \otimes\left\{\begin{array}{l}
\boldsymbol{\varepsilon}^{0(k)} \\
\boldsymbol{\kappa}^{0(k)}
\end{array}\right\}, \\
\left\{\begin{array}{l}
Q_{x} \\
Q_{y}
\end{array}\right\}=\sum_{k=1}^{n} k_{s} \mathbf{C}^{(k)} \otimes \boldsymbol{\varepsilon}_{2}^{(k)},
\end{gathered}
$$

where

$$
\begin{gathered}
\mathbf{N}=\left\{N_{x}, N_{y}, N_{x y}\right\}^{T}, \quad \mathbf{M}=\left\{M_{x}, M_{y}, M_{x y}\right\}^{T}, \\
\boldsymbol{\varepsilon}^{0(k)}=\left\{R_{1}^{(k)} \varepsilon_{x}^{0}, R_{2}^{(k)} \varepsilon_{x}^{0}, R_{3}^{(k)} \varepsilon_{x}^{0}, R_{1}^{(k)} \varepsilon_{y}^{0}, R_{2}^{(k)} \varepsilon_{y}^{0}, R_{3}^{(k)} \varepsilon_{y}^{0}, R_{1}^{(k)} \varepsilon_{x y}^{0}, R_{2}^{(k)} \varepsilon_{x y}^{0}, R_{3}^{(k)} \varepsilon_{x y}^{0}\right\}^{T}, \\
\boldsymbol{\kappa}^{0(k)}=\left\{R_{1}^{(k)} \kappa_{x}, R_{2}^{(k)} \kappa_{x}, R_{3}^{(k)} \kappa_{x}, R_{1}^{(k)} \kappa_{y}, R_{2}^{(k)} \kappa_{y}, R_{3}^{(k)} \kappa_{y}, R_{1}^{(k)} \kappa_{x y}, R_{2}^{(k)} \kappa_{x y}, R_{3}^{(k)} \kappa_{x y}\right\}^{T},
\end{gathered}
$$


and the elements in the coupled damaged stiffness tensors $\mathbf{A}^{(k)}, \mathbf{B}^{(k)}, \mathbf{D}^{(k)}$, and $\mathbf{C}^{(k)}$ are determined as

$$
\begin{aligned}
\left(A_{i j}^{(k)}, B_{i j}^{(k)}, D_{i j}^{(k)}\right) & =\int_{z_{k-1}}^{z_{k}} Q_{i j}^{d(k)}\left(1, z, z^{2}\right) \mathrm{d} z \quad(i=1,2,3, j=1,2, \ldots, 9), \\
C_{i j}^{(k)} & =\int_{z_{k-1}}^{z_{k}} Q_{i j}^{d(k)} \mathrm{d} z \quad(i=4,5, j=1,2,3,4) .
\end{aligned}
$$

Neglecting the effects of in-plane inertia, rotary inertia, and coupled normal-rotary inertia, the nonlinear equilibrium equations for moderately thick laminated plates are [21]

$$
\begin{gathered}
N_{x, x}+N_{x y, y}=0 \\
N_{x y, x}+N_{y, y}=0 \\
Q_{x, x}+Q_{y, y}+\left[N_{x} w_{, x}+N_{x y} w_{, y}\right]_{, x}+\left[N_{x y} w_{, x}+N_{y} w_{, y}\right]_{, y}=\rho_{0} h \ddot{w} \\
M_{x, x}+M_{x y, y}-Q_{x}=0 \\
M_{y, y}+M_{x y, x}-Q_{y}=0
\end{gathered}
$$

where $\rho_{0}$ is the mass of unit volume. By substituting (2.12) into (2.15), and introducing the following dimensionless parameters:

$$
\begin{gathered}
\xi=\frac{x}{a}, \quad \eta=\frac{y}{b}, \quad \lambda=\frac{a}{b}, \quad \bar{\rho}=\frac{\rho_{0} a^{2}}{t_{1}^{2} E}, \quad H=\frac{a}{h}, \quad \tau=\frac{t}{t_{1}}, \quad U=\frac{a u}{h^{2}}, \\
V=\frac{a v}{h^{2}}, \quad W=\frac{w}{h}, \quad \Lambda=H \varphi, \quad \Psi=H \psi, \quad \bar{A}_{i j}^{(k)}=\frac{A_{i j}^{(k)}}{E h}, \quad \bar{B}_{i j}^{(k)}=\frac{B_{i j}^{(k)}}{E h^{2}}, \\
\bar{D}_{i j}^{(k)}=\frac{D_{i j}^{(k)}}{E h^{3} \mathrm{~S}} \quad(i=1,2,3, j=1,2, \ldots, 9), \quad \bar{C}_{i j}^{(k)}=\frac{C_{i j}^{(k)}}{E h} \quad(i=4,5, j=1,2,3,4), \\
e_{1}^{(k)}=\frac{E_{1}^{(k)}(\tau)}{E}, \quad e_{2}^{(k)}=\frac{E_{2}^{(k)}(\tau)}{E}, \quad e_{12}^{(k)}=\frac{G_{12}^{(k)}(\tau)}{E}, \quad e_{13}^{(k)}=\frac{G_{13}^{(k)}(\tau)}{E}, \quad e_{23}^{(k)}=\frac{G_{23}^{(k)}(\tau)}{E},
\end{gathered}
$$


then, the dimensionless equilibrium equations of cross-ply laminated plates with the coupled effects of viscoelasticity and damage can be expressed as

$$
\begin{aligned}
& \sum_{k=1}^{n}\left\{\sum_{i=1}^{2} \bar{A}_{1 i}^{(k)} \otimes R_{i}^{(k)}\left(U_{, \xi \xi}+W_{, \xi} W_{, \xi \xi}\right)+\sum_{i=4}^{5} \lambda \bar{A}_{1 i}^{(k)} \otimes R_{i-3}^{(k)}\left(V_{, \xi \eta}+\lambda W_{, \eta} W_{, \xi \eta}\right)+\sum_{i=1}^{2} \bar{B}_{1 i}^{(k)} \otimes R_{i}^{(k)} \Lambda_{, \xi \xi}\right. \\
& +\lambda \sum_{i=4}^{5} \bar{B}_{1 i}^{(k)} \otimes R_{i-3}^{(k)} \Psi_{, \xi \eta}+\lambda \bar{A}_{39}^{(k)} \otimes R_{3}^{(k)}\left(\lambda U_{, \eta \eta}+V_{, \xi \eta}+\lambda W_{, \eta} W_{, \xi \eta}+\lambda W_{, \xi} W_{, \eta \eta}\right) \\
& \left.+\lambda \bar{B}_{39}^{(k)} \otimes R_{3}^{(k)}\left(\lambda \Lambda_{, \eta \eta}+\Psi_{, \xi \eta}\right)\right\}=0 \\
& \sum_{k=1}^{n}\left\{\bar{A}_{39}^{(k)} \otimes R_{3}^{(k)}\left(\lambda U_{, \xi \eta}+V_{, \xi \xi}+\lambda W_{, \eta} W_{, \xi \xi}+\lambda W_{, \xi} W_{, \xi \eta}\right)+\bar{B}_{39}^{(k)} \otimes R_{3}^{(k)}\left(\lambda \Lambda_{, \xi \eta}+\Psi_{, \xi \xi}\right)\right. \\
& +\lambda \sum_{i=1}^{2} \bar{A}_{2 i}^{(k)} \otimes R_{i}^{(k)}\left(U_{, \xi \eta}+W_{, \xi} W_{, \xi \eta}\right)+\lambda^{2} \sum_{i=4}^{5} \bar{A}_{2 i}^{(k)} \otimes R_{i-3}^{(k)}\left(V_{, \eta \eta}+\lambda W_{, \eta} W_{, \eta \eta}\right) \\
& \left.+\lambda \sum_{i=1}^{2} \bar{B}_{2 i}^{(k)} \otimes R_{i}^{(k)} \Lambda_{, \xi \eta}+\lambda^{2} \sum_{i=4}^{5} \bar{B}_{2 i}^{(k)} \otimes R_{i-3}^{(k)} \Psi_{, \eta \eta}\right\}=0 \\
& \sum_{k=1}^{n}\left\{H^{2} k_{s} \sum_{i=1}^{2} \bar{C}_{4 i}^{(k)} \otimes R_{i+3}^{(k)}\left(\Lambda_{, \xi}+W_{, \xi \xi}\right)+H^{2} \lambda k_{s} \sum_{i=3}^{4} \bar{C}_{5 i}^{(k)} \otimes R_{i+1}^{(k)}\left(\Psi_{, \eta}+\lambda W_{, \eta \eta}\right)\right. \\
& +\left[\sum_{i=1}^{2} \bar{A}_{1 i}^{(k)} \otimes R_{i}^{(k)}\left(U_{, \xi}+\frac{1}{2} W_{, \xi}^{2}\right)+\sum_{i=4}^{5} \bar{A}_{1 i}^{(k)} \otimes R_{i-3}^{(k)}\left(\lambda V_{, \eta}+\frac{\lambda^{2}}{2} W_{, \eta}^{2}\right)\right. \\
& \left.+\sum_{i=1}^{2} \bar{B}_{1 i}^{(k)} \otimes R_{i}^{(k)} \Lambda_{, \xi}+\lambda \sum_{i=4}^{5} \bar{B}_{1 i}^{(k)} \otimes R_{i-3}^{(k)} \Psi, \eta\right] W_{, \xi \xi} \\
& +2 \lambda\left[\bar{A}_{39}^{(k)} \otimes R_{3}^{(k)}\left(\lambda U_{, \eta}+V_{, \xi}+\lambda W_{, \xi} W_{, \eta}\right)+\bar{B}_{39}^{(k)} \otimes R_{3}^{(k)}\left(\lambda \Lambda_{, \eta}+\Psi_{, \xi}\right)\right] W_{, \xi \eta} \\
& +\lambda^{2}\left[\sum_{i=1}^{2} \bar{A}_{2 i}^{(k)} \otimes R_{i}^{(k)}\left(U_{, \xi}+\frac{1}{2} W_{, \xi}^{2}\right)+\sum_{i=4}^{5} \lambda \bar{A}_{2 i}^{(k)} \otimes R_{i-3}^{(k)}\left(V_{, \eta}+\frac{\lambda}{2} W_{, \eta}^{2}\right)\right. \\
& \left.\left.+\sum_{i=1}^{2} \bar{B}_{2 i}^{(k)} \otimes R_{i}^{(k)} \Lambda_{, \xi}+\lambda \sum_{i=4}^{5} \bar{B}_{2 i}^{(k)} \otimes R_{i-3}^{(k)} \Psi, \eta\right] W_{, \eta \eta}\right\}-\bar{\rho} H^{2} \ddot{W}=0, \\
& \sum_{k=1}^{n}\left\{\sum_{i=1}^{2} \bar{B}_{1 i}^{(k)} \otimes R_{i}^{(k)}\left(U_{, \xi \xi}+W_{, \xi} W_{, \xi \xi}\right)+\sum_{i=4}^{5} \lambda \bar{B}_{1 i}^{(k)} \otimes R_{i-3}^{(k)}\left(V_{, \xi \eta}+\lambda W_{, \eta} W_{, \xi \eta}\right)\right. \\
& +\sum_{i=1}^{2} \bar{D}_{1 i}^{(k)} \otimes R_{i}^{(k)} \Lambda_{, \xi \xi}+\lambda \sum_{i=4}^{5} \bar{D}_{1 i}^{(k)} \otimes R_{i-3}^{(k)} \Psi_{, \xi \eta} \\
& +\lambda \bar{B}_{39}^{(k)} \otimes R_{3}^{(k)}\left(\lambda U_{, \eta \eta}+V_{, \xi \eta}+\lambda W_{, \eta} W_{, \xi \eta}+\lambda W_{, \xi} W_{, \eta \eta}\right) \\
& \left.+\lambda \bar{D}_{39}^{(k)} \otimes R_{3}^{(k)}\left(\lambda \Lambda_{, \eta \eta}+\Psi_{, \xi \eta}\right)-H^{2} k_{s} \sum_{i=1}^{2} \bar{C}_{4 i}^{(k)} \otimes R_{i+3}^{(k)}\left(\Lambda+W_{, \xi}\right)\right\}=0,
\end{aligned}
$$




$$
\begin{aligned}
\sum_{k=1}^{n}\{ & \bar{B}_{39}^{(k)} \otimes R_{3}^{(k)}\left(\lambda U_{, \xi \eta}+V_{, \xi \xi}+\lambda W_{, \eta} W_{, \xi \xi}+\lambda W_{, \xi} W_{, \xi \eta}\right)+\bar{D}_{39}^{(k)} \otimes R_{3}^{(k)}\left(\lambda \Lambda_{, \xi \eta}+\Psi_{, \xi \xi}\right) \\
& +\lambda \sum_{i=1}^{2} \bar{B}_{2 i}^{(k)} \otimes R_{i}^{(k)}\left(U_{, \xi \eta}+W_{, \xi} W_{, \xi \eta}\right)+\lambda^{2} \sum_{i=4}^{5} \bar{B}_{2 i}^{(k)} \otimes R_{i-3}^{(k)}\left(V_{, \eta \eta}+\lambda W_{, \eta} W_{, \eta \eta}\right) \\
& \left.+\lambda \sum_{i=1}^{2} \bar{D}_{2 i}^{(k)} \otimes R_{i}^{(k)} \Lambda_{, \xi \eta}+\lambda^{2} \sum_{i=4}^{5} \bar{D}_{2 i}^{(k)} \otimes R_{i-3}^{(k)} \Psi_{, \eta \eta}-H^{2} k_{s} \sum_{i=3}^{4} \bar{C}_{5 i}^{*(k)} \otimes R_{i+1}^{(k)}\left(\Psi+\lambda W_{, \eta}\right)\right\}=0 .
\end{aligned}
$$

Suppose that all of the boundary conditions of the plate are simply supported. In such case, the dimensionless boundary conditions can be written as

$$
\begin{array}{ll}
\xi=0,1: & U=V=W=\Psi=M_{\xi \xi}=0, \\
\eta=0,1: & U=V=W=\Lambda=M_{\eta \eta}=0 .
\end{array}
$$

\section{Solution Methodology}

A solution for (2.17) in conjunction with the boundary condition (2.18) is sought in the following separable form:

$$
\begin{array}{r}
U=\sum_{m=1}^{\infty} \sum_{n=1,3, \ldots}^{\infty} f_{u}(\tau) \sin (2 \pi m \xi) \sin (\pi n \eta), \\
V=\sum_{m=1,3, \ldots}^{\infty} \sum_{n=1}^{\infty} f_{v}(\tau) \sin (\pi m \xi) \sin (2 \pi n \eta), \\
W=\sum_{m=1,3, \ldots}^{\infty} \sum_{n=1,3, \ldots}^{\infty} f_{w}(\tau) \sin (\pi m \xi) \sin (\pi n \eta), \\
\Lambda=\sum_{m=1,3, \ldots}^{\infty} \sum_{n=1,3, \ldots}^{\infty} f_{\varphi}(\tau) \cos (\pi m \xi) \sin (\pi n \eta), \\
\Psi=\sum_{m=1,3, \ldots}^{\infty} \sum_{n=1,3, \ldots}^{\infty} f_{\psi}(\tau) \sin (\pi m \xi) \cos (\pi n \eta) .
\end{array}
$$

Substituting (3.1) into the governing equations (2.17) and making use of the oneterm approximation of the Galerkin method, we can transform the nonlinear integral-partial differential equations into the nonlinear integral-ordinary differential equations in terms of $f_{u}(\tau), f_{v}(\tau), f_{w}(\tau), f_{\varphi}(\tau)$, and $f_{\psi}(\tau)$. The domain is divided by square mesh into $M \times M$ divisions and the time $\tau$ is equally divided into small time segments $\Delta \tau$. The Simpson integral formula is used to compute the integrations with respect to the spatial coordinates and the Newton-Cotes trapezoidal rule is used to compute the integrations with respect to time. 
Moreover, using the Newmark scheme, the acceleration item $\ddot{f}_{w}$ and velocity item $\dot{f}_{w}$ can be expressed as follows:

$$
\begin{gathered}
\ddot{f}_{w}^{(N)}=\frac{4\left(f_{w}^{(N)}-f_{w}^{(N-1)}\right)}{(\Delta \tau)^{2}}-\frac{4 \dot{f}_{w}^{(N-1)}}{\Delta \tau}-\ddot{f}_{w}^{(N-1)}, \\
\dot{f}_{w}^{(N)}=\dot{f}_{w}^{(N-1)}+\frac{1}{2}\left[\ddot{f}_{w}^{(N-1)}+\ddot{f}_{w}^{(N)}\right] \Delta \tau,
\end{gathered}
$$

where $f_{w}^{(N)}$ denotes the value of $f_{w}(\tau)$ at the time $N \Delta \tau$. Through these treatings, (2.17) can be transformed into the nonlinear algebraic equations only with time. And the whole equations are iterated to seek solutions. At each step of the iteration, the nonlinear items are linearized. For example, at the step $J$, the nonlinear items may be transformed to

$$
(X \cdot Y)_{J}=(X)_{J} \cdot(Y)_{J_{P}}
$$

where $(Y)_{J_{P}}$ is the average value of those obtained in the preceding two iterations. For the initial step of the iteration, it can be determined by using the quadratic extrapolation, that is,

$$
(Y)_{J_{P}}=A A(Y)_{J-1}+B B(Y)_{J-2}+C C(Y)_{J-3}
$$

And for the different step of the iteration, the coefficients $A A, B B$, and $C C$ can be expressed as follow:

$$
\begin{aligned}
& J=1: \quad A A=1, \quad B B=0, \quad C C=0, \\
& J=2: \quad A A=2, \quad B B=-1, \quad C C=0, \\
& J \geq 3: \quad A A=3, \quad B B=-3, \quad C C=1 .
\end{aligned}
$$

For every time step, the iteration lasts until the difference of the present value and the former is smaller than $0.1 \%$; then continue the calculation of the next step.

\section{Damage Evolution Equation}

In the present research, the following damage evolution equation is employed [19, 22]:

$$
\frac{\mathrm{d} D_{i}^{(k)}}{\mathrm{d} \tau}=\left\{\begin{array}{ll}
B_{i}\left(\frac{\sigma_{i}^{(k)}}{1-D_{i}^{(k)}}\right)^{m_{i}}, & \sigma_{i}^{(k)} \geq \sigma_{D_{i}}^{(k)}, \quad \\
0, & \sigma_{i}^{(k)}<\sigma_{D_{i}}^{(k)} .
\end{array} \quad(i=1,2)\right.
$$


Table 1: Comparison of fundamental linear frequencies of laminated elastic plates $(a / h=10)$.

\begin{tabular}{lcccc}
\hline $\begin{array}{l}\text { Reference [23] } \\
\text { SOT }\end{array}$ & CPT & Reference [24] & TOT & Present \\
\hline 12.527 & 15.104 & 12.163 & 11.958 & FOT \\
\hline
\end{tabular}

Here $B_{i}$ and $m_{i}$ are the material constants. $\sigma_{D_{i}}^{(k)}$ is the stress threshold value in the $i$ direction at which the damage $D_{i}^{(k)}$ begins to grow, and

$$
\sigma_{1}^{(k)}=\frac{1}{h^{(k)}} \int_{z_{k-1}}^{z_{k}} \tilde{\sigma}_{x}^{(k)} \mathrm{d} z, \quad \sigma_{2}^{(k)}=\frac{1}{h^{(k)}} \int_{z_{k-1}}^{z_{k}} \tilde{\sigma}_{y}^{(k)} \mathrm{d} z
$$

Assume that there is no damage at initial time; thus, the damage values of all points are $D_{1}^{(k)}(0)=0$ and $D_{2}^{(k)}(0)=0$. When stopping iterative step $J$, we can obtain the five function values $f_{u}(\tau), f_{v}(\tau), f_{w}(\tau), f_{\varphi}(\tau)$, and $f_{\psi}(\tau)$. Before continuing the next iterative step, it must determine whether the damage values of an arbitrary point of the plate develop. Therefore, firstly, the displacements of an arbitrary point of the plate can be obtained by using (3.1). Calculate the strains of an arbitrary point of the plate by using (2.2) and (2.3). Then, using (2.5), (2.8) and (4.2), $\sigma_{1}^{(k)}$ and $\sigma_{2}^{(k)}$ can be obtained. Finally, use (4.1) to determine whether the damage grows. Suppose that $D_{i}^{(k)}(J)$ is the damage value of an arbitrary point for the $k$ th layer in the $i$ direction at the time $J \Delta \tau$. If $\sigma_{i}^{(k)} \geq \sigma_{D_{i}}^{(k)}$, then the damage value at the time $J \Delta \tau$ is

$$
D_{i}^{(k)}(J)=D_{i}^{(k)}(J-1)+\dot{D}_{i}^{(k)}\left(D_{i}^{(k)}(J-1), \sigma_{i}^{(k)}\right) \Delta \tau
$$

and if $\sigma_{i}{ }^{(k)}<\sigma_{D_{i}}^{(k)}$, then

$$
D_{i}^{(k)}(J)=D_{i}^{(k)}(J-1)
$$

\section{Numerical Results}

To ensure the accuracy and effectiveness of the present method, the fundamental frequencies of a three-layer symmetric cross-ply $\left[0^{\circ} / 90^{\circ} / 0^{\circ}\right]$ laminated elastic plate without considering damage effect are solved firstly and the materials properties $[23,24]$ are given as $E_{1}=25 E_{2}$, $G_{12}=G_{13}=0.5 E_{2}, G_{23}=0.2 E_{2}$, and $\nu_{12}=0.25$. Define $\bar{\omega}_{0}=\left(\omega_{0} a^{2} / h\right) \sqrt{\rho_{0} / E_{2}}$, in which $\bar{\omega}_{0}$ and $\omega_{0}$ are the dimensionless and dimensional linear frequencies of laminated elastic plates without damage, respectively. The fundamental frequencies are calculated and compared in Table 1 with those of $[23,24]$. Table 1 shows that the present result approximately agrees with the result in [24] by the first-order shear-deformation theory (FOT). And for the moderately thick laminated plates, the FOT solution has small difference compared with the second-order shear-deformation theory (SOT) solution and third-order shear-deformation theory (TOT) solution, but large difference is compared with the classical plate theory (CPT). It is noted that the effect of the transverse shear deformation cannot be neglected for the moderately thick laminated plates. 
Table 2: Effect of transverse shear on the nonlinear vibrating frequency $\Omega^{*}$ of laminated plates.

\begin{tabular}{|c|c|c|c|c|c|}
\hline \multirow{2}{*}{$w_{\max } / h$} & \multirow{2}{*}{ Case } & \multicolumn{4}{|c|}{$a / h$} \\
\hline & & 5 & 10 & 15 & 20 \\
\hline \multirow{4}{*}{1} & $T_{s}=0, T_{d}=0$ & 2.73182 & 1.33970 & 0.883711 & 0.677714 \\
\hline & $T_{s}=0, T_{d}=1$ & 2.38090 & 1.30900 & 0.872317 & 0.670063 \\
\hline & $T_{s}=1, T_{d}=0$ & 2.15917 & 1.21767 & 0.847933 & 0.649089 \\
\hline & $T_{s}=1, T_{d}=1$ & 1.90400 & 1.20830 & 0.840535 & 0.64502 \\
\hline \multirow{4}{*}{2} & $T_{s}=0, T_{d}=0$ & 3.92699 & 2.02683 & 1.36591 & 0.998917 \\
\hline & $T_{s}=0, T_{d}=1$ & 2.84119 & 1.74745 & 1.30736 & 0.991473 \\
\hline & $T_{s}=1, T_{d}=0$ & 3.69599 & 1.92329 & 1.32000 & 0.987922 \\
\hline & $T_{s}=1, T_{d}=1$ & 2.68778 & 1.65347 & 1.27967 & 0.981748 \\
\hline
\end{tabular}

Consider the amplitudes and the frequencies varying with time and the viscoelasticity and damage developing with the increase of time, so we must elect the later vibrating period to be analyzed. Hence, the relationship of the average frequency and the maximum amplitude in the 20th period are presented. Also, assume that every layer has the same material and the identical thickness. The parameters used in numerical calculation are taken as

$$
\begin{gathered}
e_{1}=9.75+0.25 e^{-\alpha_{1} \tau}, \quad \alpha_{1}=0.022, \\
e_{2}=0.32+0.68 e^{-\alpha_{2} \tau}, \quad \alpha_{2}=0.024, \quad v_{12}=0.4, \\
e_{12}=0.07+0.16 e^{-\alpha_{12} \tau}, \quad \alpha_{12}=0.026, \\
e_{13}=0.07+0.16 e^{-\alpha_{13} \tau}, \quad \alpha_{13}=0.026, \\
e_{23}=0.05+0.12 e^{-\alpha_{23} \tau}, \quad \alpha_{23}=0.026, \\
B_{1}=3.7 \times 10^{-12} \mathrm{MPa}^{-m_{1}}, \quad B_{2}=4.8 \times 10^{-11} \mathrm{MPa}^{-m_{2}}, \quad m_{1}=m_{2}=3 .
\end{gathered}
$$

Define

$$
\Omega^{*}=\Omega \sqrt{\frac{\rho_{0} a^{2}}{E}}
$$

where $\Omega^{*}$ and $\Omega$ are the dimensionless and dimensional nonlinear free vibrating frequency of viscoelastic plates with damage, respectively.

Table 2 shows the effect of transverse shear deformation on the nonlinear free vibrating frequency of the viscoelastic cross-ply $\left[0^{\circ} / 90^{\circ} / 0^{\circ}\right]$ laminated square plate with considering damage effect or without considering damage effect, in which $w_{\max } / h$ is the dimensionless maximum vibration amplitude of the center point of the plate (similarly below), $T_{S}$ is the tracing constant which represents the influence of transverse shear when $T_{s}=1$ and the effect is neglected when $T_{s}=0$, as well as $T_{d}$ is the tracing constant which represents the influence of damage when $T_{d}=1$ and the effect is neglected when $T_{d}=0$. From Table 2 it may be observed that the thicker the thickness of the laminated plate, the more significant the effect of transverse shear on the nonlinear free vibrating frequency of the laminated plate. And in all cases transverse shear results in a decrease of the vibrating frequency. Therefore, the effect of transverse shear deformation is considered in the following calculated examples. 


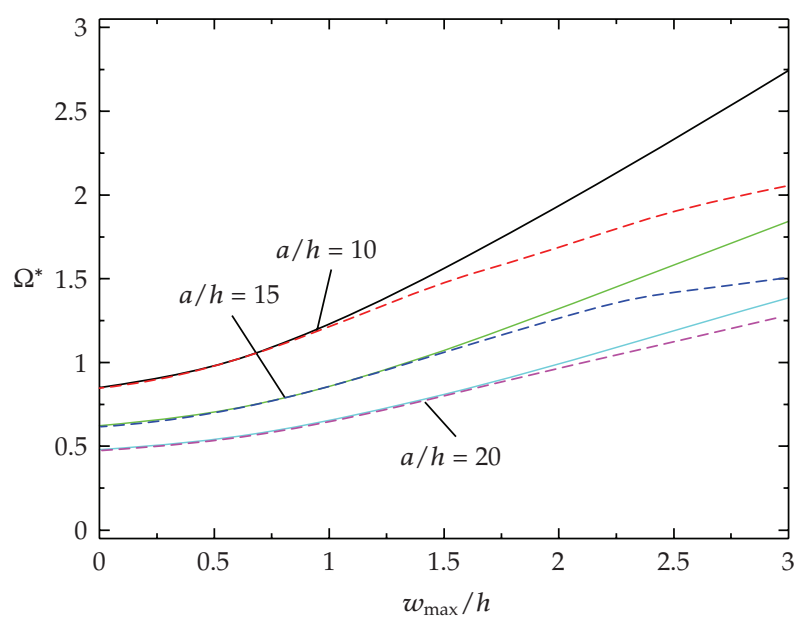

Figure 1: Effect of span-thickness ratio on the nonlinear amplitude-frequency curves of viscoelastic laminated plate.

Figure 1 shows the effect of span-thickness ratio $a / h$ on the nonlinear free vibrating amplitude-frequency response curves of the viscoelastic cross-ply $\left[0^{\circ} / 90^{\circ} / 0^{\circ}\right]$ laminated square plate. In Figure 1, the solid line denotes the undamaged condition, while the dashed line denotes the condition with damage evolution. It can be observed that with the decrease of the span-thickness ratio, namely, the increase of thickness of the plate, the nonlinear free vibrating frequency of the plate becomes bigger under a given vibration amplitude. Meanwhile, the frequency increases with increase of the vibration amplitude under a fixed span-thickness ratio. It can be also seen that the two curves with/without damage agree very well where the vibration amplitude is small. But with the increase of the vibration amplitude, the damage begins to appear and the accumulation velocity of the damage increases, which results in the stiffness of the plate becoming smaller. Accordingly, the nonlinear vibration frequency considering damage reduces remarkably than the result neglecting damage. Moreover, the less the span-thickness ratio of the plate is, the more the damage becomes, then the more the frequency descends.

Figure 2 displays the effect of aspect ratio $\lambda$ on the nonlinear free vibrating amplitudefrequency response curves of the viscoelastic cross-ply $\left[0^{\circ} / 90^{\circ} / 0^{\circ}\right]$ laminated plate. With the increase of the aspect ratio, that is, the width decreases under the condition that the length remains constant, the nonlinear free vibrating frequency of the plate becomes bigger. Similarly, under the given aspect ratio $\lambda$, the increase of the amplitude will expedite the accumulation velocity of the damage which will cause the more reduction in the stiffness of the plate. Consequently, the reduction of frequency caused by the damage will be more remarkable. The more the aspect ratio is, the more the frequency reduces.

The effect of material viscoelasticity parameter $\alpha\left(=\alpha_{1}=\alpha_{2}=\alpha_{12}=\alpha_{13}=\alpha_{23}\right)$ on the nonlinear free vibrating frequency of the viscoelastic cross-ply $\left[0^{\circ} / 90^{\circ} / 0^{\circ}\right]$ laminated square plate is listed in Table 3. The nonlinear free vibrating frequency of the laminated plate decreases with the higher value of the material viscoelasticity parameter. Due to the fact that viscoelastic material possesses dissipative nature and it acts as damping in the dynamic problems, it can improve the stability of the structure. Therefore, at the same condition, the higher viscoelasticity parameter can suppress the emergence of damage and 


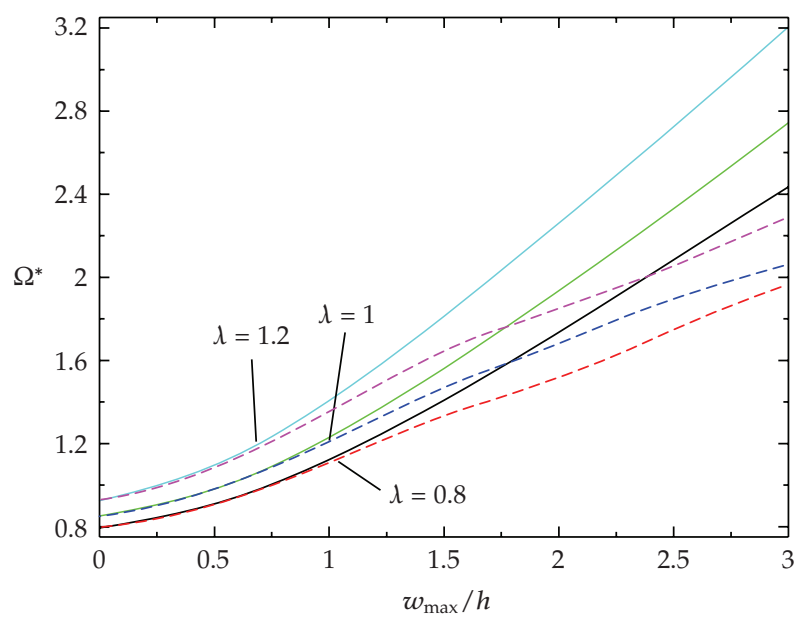

Figure 2: Effect of aspect ratio on the nonlinear amplitude-frequency curves of viscoelastic laminated plate $(a / h=10)$.

Table 3: Effect of viscoelasticity parameter on the nonlinear vibrating frequency $\Omega^{*}$ of laminated plates $(a / h=15)$.

\begin{tabular}{|c|c|c|c|c|c|c|}
\hline \multirow{2}{*}{$\alpha$} & \multirow{2}{*}{ Case } & \multicolumn{5}{|c|}{$w_{\max } / h$} \\
\hline & & 1 & 1.5 & 2 & 2.5 & 3 \\
\hline \multirow{2}{*}{0} & $T_{d}=0$ & 0.850871 & 1.07477 & 1.35062 & 1.61494 & 1.85181 \\
\hline & $T_{d}=1$ & 0.837562 & 1.04704 & 1.28289 & 1.46316 & 1.49701 \\
\hline \multirow{2}{*}{0.5} & $T_{d}=0$ & 0.846744 & 1.06551 & 1.31475 & 1.56880 & 1.84306 \\
\hline & $T_{d}=1$ & 0.838762 & 1.05061 & 1.25715 & 1.42693 & 1.49761 \\
\hline \multirow{2}{*}{2} & $T_{d}=0$ & 0.820509 & 1.04718 & 1.28120 & 1.53075 & 1.79590 \\
\hline & $T_{d}=1$ & 0.820229 & 1.04668 & 1.25565 & 1.42815 & 1.47021 \\
\hline
\end{tabular}

cause the difference of the vibrating frequency between the damaged plate and undamaged plate decrease.

\section{Conclusions}

The nonlinear free vibration for viscoelastic cross-ply laminated composite plates under considering transverse shear deformation and damage effect has been investigated. The effects of transverse shear, material viscoelasticity, span-thickness ratio, aspect ratio, and damage effect on the nonlinear vibration of laminated plates have been discussed. In summary, the transverse shear effect on the nonlinear vibration of the viscoelastic laminated plates with damage is significant, especially, for the laminates with large thickness. This effect decreases the nonlinear frequency but does not change the general behavior of the nonlinear vibration in all cases. With the decrease of span-thickness ratio or increase of aspect ratio, the vibrating frequency increases, as well as the difference of vibration frequency between considering damage and neglecting damage becomes bigger. In addition, with the increase of the vibration amplitude, the damage begins to appear, the accumulation velocity of the damage increases, and the reduction of the vibrating frequency becomes more significant. The larger the material viscoelasticity, the smaller the reduction of the vibrating frequency. 


\section{Acknowledgments}

The project was supported by the Young Technological Talents' Innovation Foundation of Fujian Province (2006F3077) and Scientific and Technical Development Foundation of Fuzhou University (2007-XQ-20).

\section{References}

[1] J. Aboudi and G. Cederbaum, "Dynamic stability analysis of viscoelastic plates by Lyapunov exponents," Journal of Sound and Vibration, vol. 139, no. 3, pp. 459-467, 1990.

[2] L. Librescu and N. K. Chandiramani, "Dynamic stability of transversely isotropic viscoelastic plates," Journal of Sound and Vibration, vol. 130, no. 3, pp. 467-486, 1989.

[3] Y. X. Sun and S. Y. Zhang, "Chaotic dynamic analysis of viscoelastic plates," International Journal of Mechanical Sciences, vol. 43, no. 5, pp. 1195-1208, 2001.

[4] L. Q. Chen, J. W. Zu, and J. Wu, "Steady-state response of the parametrically excited axially moving string constituted by the Boltzmann superposition principle," Acta Mechanica, vol. 162, no. 1-4, pp. 143-155, 2003.

[5] T. W. Kim and J. H. Kim, "Nonlinear vibration of viscoelastic laminated composite plates," International Journal of Solids and Structures, vol. 39, no. 10, pp. 2857-2870, 2002.

[6] S. C. Yu and S. C. Huang, "Vibration of a three-layered viscoelastic sandwich circular plate," International Journal of Mechanical Sciences, vol. 43, no. 10, pp. 2215-2236, 2001.

[7] D. L. Prabhakara and P. K. Datta, "Vibration and static stability characteristics of rectangular plates with a localized flaw," Computers and Structures, vol. 49, no. 5, pp. 825-836, 1993.

[8] D. L. Prabhakara and P. K. Datta, "Parametric instability characteristics of rectangular plates with localized damage subjected to in-plane periodic load," Structural Engineering Review, vol. 1, pp. 7179, 1993.

[9] P. A. A. Laura and R. H. Gutierrez, "Transverse vibration of a damaged circular annular plate with a free edge," Journal of Sound and Vibration, vol. 241, no. 4, pp. 720-724, 2001.

[10] D. F. Sheng and C. J. Cheng, "Dynamical behaviors of nonlinear viscoelastic thick plates with damage," International Journal of Solids and Structures, vol. 41, no. 26, pp. 7287-7308, 2004.

[11] Y. Fu, P. Li, and Y. Zheng, "Analysis of nonlinear dynamic response for viscoelastic composite plate with transverse matrix cracks," Acta Mechanica Solida Sinica, vol. 17, no. 3, pp. 230-238, 2004.

[12] Y. Fu and Y. H. Lu, "Analysis of the nonlinear dynamic response of viscoelastic symmetric cross-ply laminated plates with transverse matrix crack," Composite Structures, vol. 72, no. 4, pp. 469-476, 2006.

[13] Y. F. Zheng and Y. Fu, "Effect of damage on bifurcation and chaos of viscoelastic plates," International Journal of Nonlinear Sciences and Numerical Simulation, vol. 6, no. 1, pp. 87-92, 2005.

[14] Y. F. Zheng and Y. Fu, "Effect of damage on nonlinear dynamic properties of viscoelastic rectangular plates," Applied Mathematics and Mechanics, vol. 26, no. 3, pp. 319-326, 2005.

[15] Y. F. Zheng and Y. Fu, "Nonlinear dynamic analysis of viscoelastic/damage behaviour for symmetric cross-ply laminated plates," Advances in Vibration Engineering, vol. 3, pp. 185-197, 2004.

[16] Y. F. Zheng and Y. Fu, "Analysis of nonlinear vibration for symmetric angle-ply laminated viscoelastic plates with damage," Acta Mechanica Sinica, vol. 21, no. 5, pp. 459-466, 2005.

[17] R. D. Mindlin, "Influences of rotatory inertia and shear inflexural motion of isotropic, elastic plates," Journal of Applied Mechanics, vol. 18, pp. 1031-1036, 1951.

[18] C. L. Chow and J. Wang, "An anisotropic theory of elasticity for continuum damage mechanics," International Journal of Fracture, vol. 33, no. 1, pp. 3-16, 1987.

[19] L. M. Kachanov, Introduction to Continuum Damage Mechanics, Martinus Nijhoff, Dordrecht, The Netherlands, 1986.

[20] A. C. Pipkin, Lectures on Viscoelasticity Theory, Springer, New York, NY, USA, 1986.

[21] Y. Nath and K. K. Shukla, "Non-linear transient analysis of moderately thick laminated composite plates," Journal of Sound and Vibration, vol. 247, no. 3, pp. 509-526, 2001.

[22] W. H. Zhang, Numerical analysis of continuum damage mechanics, Ph.D. thesis, University of New South Wales, Sydney, Australia, 1992. 
[23] A. A. Khdeir and J. N. Reddy, "Free vibrations of laminated composite plates using second-order shear deformation theory," Computers and Structures, vol. 71, no. 6, pp. 617-626, 1999.

[24] L. Librescu, A. A. Khdeir, and D. Frederick, "A shear deformable theory of laminated composite shallow shell-type panels and their response analysis I: free vibration and buckling," Acta Mechanica, vol. 76, no. 1-2, pp. 1-33, 1989. 


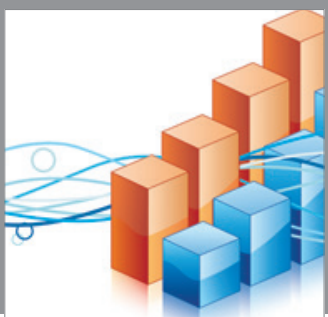

Advances in

Operations Research

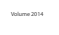

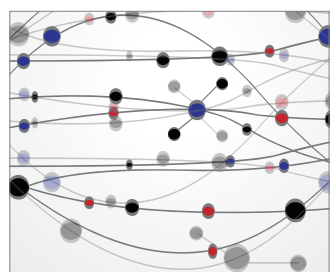

\section{The Scientific} World Journal
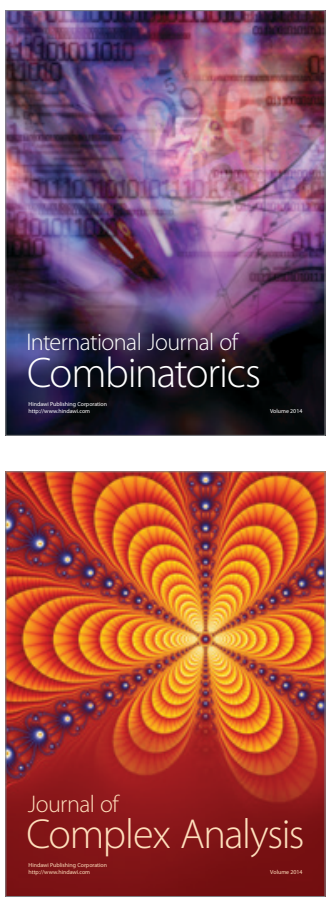

International Journal of

Mathematics and

Mathematical

Sciences
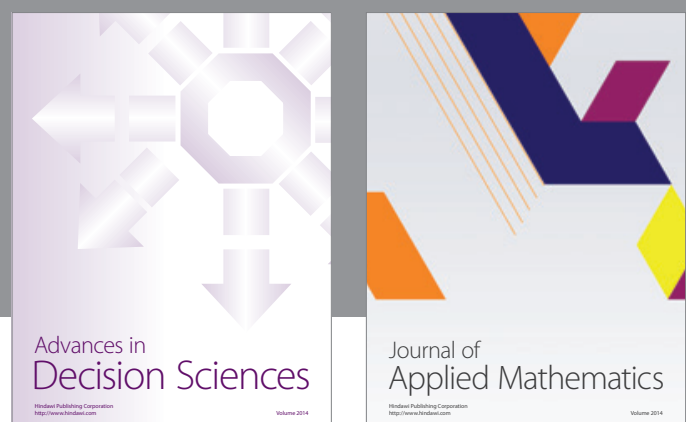

Journal of

Applied Mathematics
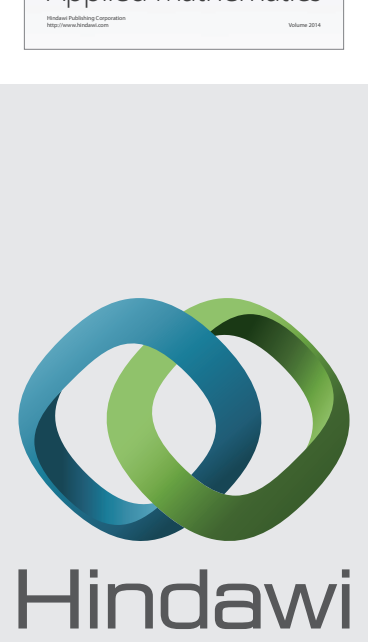

Submit your manuscripts at http://www.hindawi.com
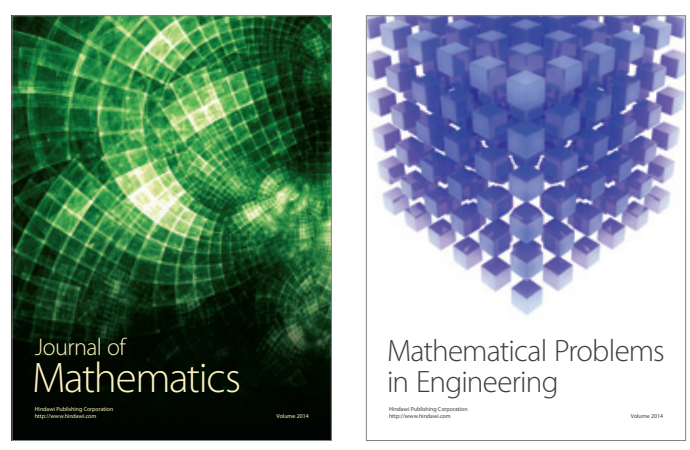

Mathematical Problems in Engineering
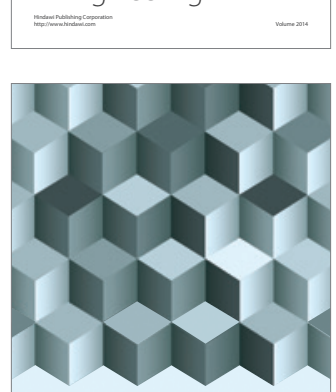

Journal of

Function Spaces
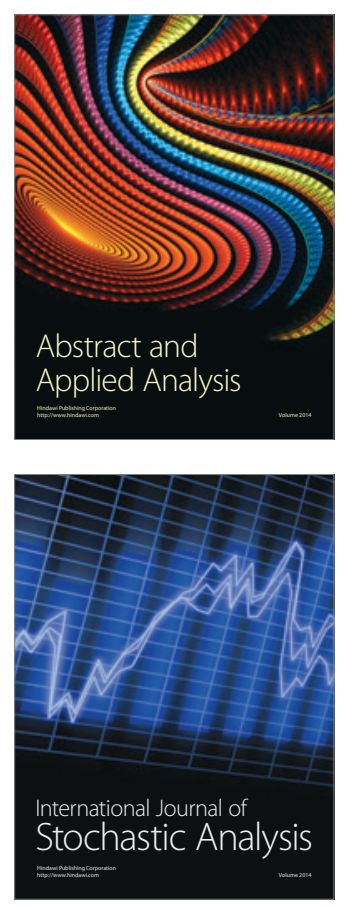



ournal of

Probability and Statistics

Promensencen
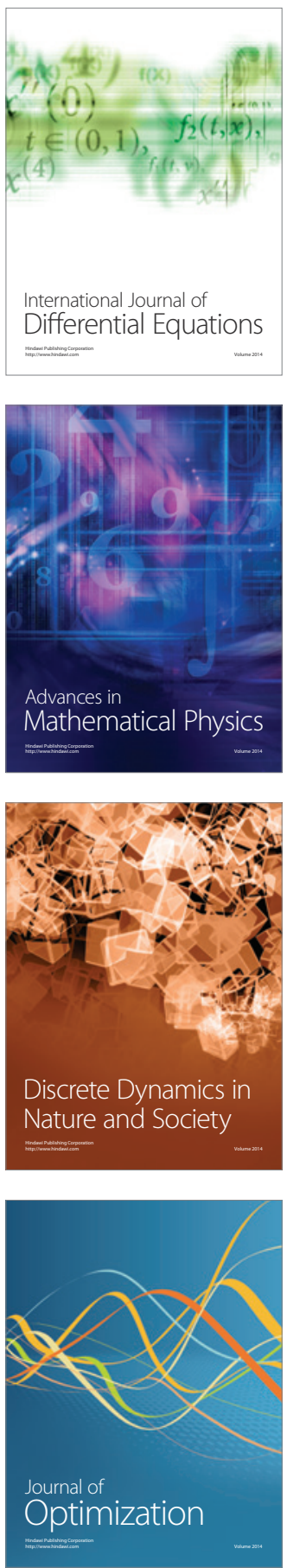